\title{
Impact of stereochemistry on biological effects of permethrin: induction of apoptosis in human hepatoma cells (HCC-I.2) and primary rat hepatocyte cultures
}

\author{
Julia Dornetshuber ${ }^{1}$, Wolfgang Bicker ${ }^{2}$, Michael Lämmerhofer ${ }^{2}$, \\ Wolfgang Lindner ${ }^{2}$, Anneliese Karwan ${ }^{1}$ and Wilfried Bursch*1
}

\author{
Address: ${ }^{1}$ Department of Medicine I, Institute of Cancer Research, Research Unit Toxicology and Prevention, Medical University of Vienna, Austria \\ and 2 Institute of Analytical Chemistry and Food Chemistry, University of Vienna, Austria \\ Email: Wilfried Bursch* - wilfried.bursch@meduniwien.ac.at \\ * Corresponding author
}

from 13th Scientific Symposium of the Austrian Pharmacological Society (APHAR). Joint Meeting with the Austrian Society of Toxicology (ASTOX) and the Hungarian Society for Experimental and Clinical Pharmacology (MFT)

Vienna, Austria. 22-24 November 2007

Published: 14 November 2007

BMC Pharmacology 2007, 7(Suppl 2):A65 doi:10.1 I86/|47|-22 I0-7-S2-A65

This abstract is available from: http://www.biomedcentral.com/I47I-22I0/7/S2/A65

(c) 2007 Dornetshuber et al; licensee BioMed Central Ltd.

Stereochemistry plays a crucial role in determining the toxicological profile of many chiral xenobiotics, e.g. the insecticidal action of mixtures containing the four stereoisomers of permethrin is essentially brought about by the $(1 R, c i s)$ - and $(1 R$, trans)-forms. Primarily non-ion channel related mammalian effects like induction of cytochrome P450 enzymes and inhibition of mitochondrial complex I - relevant endpoints in elucidating a chemical's mode of action and thus toxicological risk assessment were elucidated in studies with four-isomer mixtures of permethrin only [1-3]. Therefore, we initiated a project to shed light on the stereoselectivity of permethrin effects in mammals, using human hepatoma cells (HCC-1.2) and primary rat hepatocyte cultures as test models. Here we report (1) a commercially available four-isomer mixture of permethrin (cis-racemate/trans-racemate 25:75) exhibited a dose-dependent $(2-50 \mu \mathrm{M})$ pro-apoptotic activity; (2) the physiological death signal TGF- $\beta 1$ (10 ng/ $\mathrm{ml}$ ) and permethrin exerted an additive pro-apoptotic effect; (3) purified permethrin stereoisomers, i.e. $(1 R, c i s)$, (1S, cis), $(1 R$, trans $),(1 S$, trans $)$, exhibited - in contrast to their insecticidal action - no significant differences in their pro-apoptotic action as compared to the four-isomer mixture; (4) the pro-apoptotic potency of permethrin was lost upon metabolism to permethrinic acid, 3-phenoxybenzyl alcohol, and 3-phenoxybenzoic acid.

\section{References}

I. Gassner B, Wüthrich A, Scholtysik G, Solioz M: The pyrethroids permethrin and cyhalothrin are potent inhibitors of the mitochondrial complex I. J Pharmacol Exp Ther 1997, 28I:855-860.

2. Kostka G, Palut D, Kopeć-Szlezak J, Ludwicki JK: Early hepatic changes in rats induced by permethrin in comparison with DDT. Toxicology 2000, I42:135-143.

3. Heder AF, Hirsch-Ernst KI, Bauer D, Kahl GF, Desel H: Induction of cytochrome $\mathrm{P} 450$ 2B I by pyrethroids in primary rat hepatocyte cultures. Biochem Pharmacol 200I, 62:71-79. 